\title{
Virtual Reality as a Context for Adaptation
}

\author{
Mathew Yarossi ${ }^{1,2 *}$, Madhur Mangalam ${ }^{1}$, Stephanie Naufel ${ }^{3}$ and Eugene Tunik ${ }^{1,2,4}$ \\ ${ }^{1}$ Department of Physical Therapy, Movement and Rehabilitation Sciences, Northeastern University, Boston, MA, United States, \\ ${ }^{2}$ Department of Electrical and Computer Engineering, Northeastern University, Boston, MA, United States, ${ }^{3}$ Facebook Reality \\ Labs Research, Menlo Park, CA, United States, ${ }^{4}$ Department of Bioengineering, Northeastern University, Boston, MA, \\ United States
}

The COVID-19 pandemic has accelerated interest in virtual reality (VR) for education, entertainment, telerehabilitation, and skills training. As the frequency and duration of VR engagement increases - the number of people in the United States using VR at least once per month is forecasted to exceed 95 million-it is critical to understand how VR engagement influences brain and behavior. Here, we evaluate neurophysiological effects of sensory conflicts induced by VR engagement and posit an intriguing hypothesis: the brain processes VR as a unique "context" leading to the formation and maintenance of independent sensorimotor representations. We discuss known VRinduced sensorimotor adaptations to illustrate how VR might manifest as a context for learning and how technological and human factors might mediate the contextdependency of sensorimotor representations learned in VR.

\section{OPEN ACCESS}

Edited by:

Nadia Magnenat Thalmann, Université de Genève, Switzerland

Reviewed by:

David Harris,

University of Exeter, United Kingdom Richard Skarbez,

La Trobe University, Australia

*Correspondence:

Mathew Yarossi

m.yarossi@northeastern.edu

Specialty section: This article was submitted to Virtual Reality and Human Behaviour, a section of the journal

Frontiers in Virtual Reality

Received: 29 June 2021 Accepted: 23 September 2021 Published: 18 November 2021

Citation:

Yarossi M, Mangalam M, Naufel S and Tunik E (2021) Virtual Reality as a

Context for Adaptation.

Front. Virtual Real. 2:733076. doi: $10.3389 /$ frvir.2021.733076
Keywords: virtual reality, context learning, vestibulo-ocular reflex, optic flow, motor learning, sensorimotor transformation

\section{INTRODUCTION}

The COVID-19 pandemic has accelerated interest in virtual reality (VR) for education (Affouneh et al., 2020; Pears et al., 2020; Pregowska et al., 2021), entertainment (Sigala, 2020), telerehabilitation (Mantovani et al., 2020; Singh et al., 2020; Wang et al., 2020), and skills training (De Ponti et al., 2020; Ehrlich et al., 2020). As the frequency and duration of VR engagement increases-the number of people in the United States using VR at least once per month is forecasted to exceed 95 million (Vailshery, 2021) - it is important to understand how VR engagement influences the brain and behavior. Here, we evaluate the known behavioral and neurophysiological effects of sensory conflicts such as visual-vestibular mismatch induced by VR engagement and posit an alluring hypothesis: the brain interprets VR as a unique "context", leading to the formation and maintenance of specific sensorimotor representations for VR engagement. We provide a working definition of VR as a context and offer examples of how context-specificity of VR may influence the brain and behavior at different levels of sensorimotor functioning: vestibulo-ocular reflex (VOR) gains, visuomotor adaptation of voluntary movements, and spatial navigation. We next review several technological and human factors that may influence the extent to which the brain might interpret VR as a unique context for learning and performance. Finally, we identify the implications of this hypothesis and avenues for additional scientific exploration.

What is a context for learning? Two definitions of "context" are relevant to VR. In experimental psychology (associative learning (Aiba et al., 1994; Wasserman and Miller, 1997; Bouton, 2010; Rosas et al., 2013; Urcelay and Miller, 2014), fear conditioning (Antoniadis and McDonald, 2000; Marschner et al., 2008; Maren et al., 2013), semantic memory (Kutas and Federmeier, 2000; 
Federmeier et al., 2002; Jones et al., 2015)), context-specificity implies that a behavior is more likely to be displayed in the state, place, or circumstance in which it was learned (e.g., attending an examination in the same location as the place of study results in better retrieval of the subject matter). In sensorimotor neuroscience, context-dependent adaptation refers to learning multiple motor programs depending on specific sensory conditions and efficiently retrieving the learned motor programs later upon recognition of the same sensory conditions (Glover and Dixon, 2001; Richter et al., 2004; Burguiere et al., 2005; Welch and Ting, 2014; Neszmélyi and Horváth, 2019). Here, we define a "VR context" as a set of sensory cues associated with engagement with immersive head-mounted display-based virtual reality (HMD-VR), and "context-dependent learning" as the memory of learned adaptations that previously yielded reduced sensory conflict and hence more accurate behavior in VR. In this perspective, we focus our attention on the context-dependencies of the VR experience agnostic to the virtual scene, task, or paradigm. We, therefore, focus more directly on the sensorimotor aspects of the "VR context." However, we do not exclude the possibility of contextdependent behavioral patterns associated with the content of the virtual scene.

\section{How Might VR Manifest as a Context?}

Repeated experiences within a context can enhance retrieval of specific adaptation strategies required for successful actions. For example, with repeated exposures to VR, a user may over time form a prediction about a sensorimotor error experienced in VR. Donning a head-mounted display (HMD) may cue recall of a previously learned adaptation strategy to overcome the error, establishing VR as the context for retrieval of previous learning. This context-specific learning may involve simple reflex adaption, visuomotor adaptation of voluntary movements, and navigationbased adaptations.

\subsection{VR as a Context for Reflex Adaptation}

Relatively low-tech experiences, such as wearing corrective magnifying lenses or scuba goggles, provide clues about context-dependent learning during engagement with HMDVR. These accessories alter the perceived distance, position, and size of objects, creating a vestibular-ocular conflict akin to that experienced in VR and requiring recalibration of the vestibulo-ocular reflex (VOR) to stabilize gaze. VOR is a low latency (10-12 ms) reflex that enables eye rotation in an equal and opposite direction of head rotation to maintain gaze fixation (Gauthier and Robinson, 1975; Gonshor and Jones, 1976a; Gonshor and Jones, 1976b; Paige and Sargent, 1991). Atypical viewing conditions can result in the loss of fixation due to insufficient ocular compensation for head rotation. Image blur due to this "retinal slip" serves as an error signal, encouraging the adaptation of the VOR gain to minimize the blur (Ito, 1998). Multiple VOR gains can be toggled as appropriate contexts arise. For instance, donning a familiar pair of magnifying eyeglasses induces rapid changes in VOR gain to accommodate the magnification (Collewijn et al., 1983; Demer et al., 1987). Simply, the tactile feedback of putting on or even touching scuba goggles suffices to toggle VOR adaptation in experienced divers (Virre, 1996; Sharoni et al., 2001). Does VR also constitute a context for which a VOR gain is learned and retrieved under specific sensory conditions?

Visuovestibular conflict induced by dynamic head-tracking errors and delays in virtual environment projection result in a velocity-dependent phase lag between the vestibular feedback of head rotation and visual feedback of scene rotation (DiZio and Lackner, 1992). Just like corrective magnifying lenses, visuovestibular conflict in VR also induces VOR adaptation (Draper, 1996, 1998; Virre, 1996). For example, reduced VOR gain was found following $20 \mathrm{~min}$ of gameplay when head rotation was used to direct the character's movement and returned to normal $30 \mathrm{~min}$ following cessation of VR engagement (Di Girolamo et al., 2001). In a cohort of patients with unilateral vestibular hypofunction, VOR gain increased following 1 month of vestibular training using a VR racing game (Micarelli et al., 2017, 2019). This cohort also showed better retention of increased VOR gain at a 12-month follow-up than a comparable cohort that received conventional vestibular training alone (Viziano et al., 2019). More investigations of VOR adaptation in healthy individuals using modern HMD-VR systems with repeated engagements are needed to comprehensively probe these phenomena.

Given these initial studies, we hypothesize that VR may constitute a context for which VOR gain can be learned and retrieved whenever that context is recalled based on sensory cues (Figure 1). Similar to how putting on goggles can retrieve VOR adaptation specific to the lenses' magnification (Herdman, 1998; Gimmon et al., 2018), donning an HMD may also drive retrieval of a learned VOR adaptation. Contextual cues typically associated with VOR adaptation such as vergence angle (Lewis et al., 2003), head position (Tan et al., 1992; Yakushin et al., 2003), and eye position (Shelhamer et al., 1992) are common attributes of HMD-VR headsets (Kramida, 2015), and hence, VOR may adapt specifically to HMD-VR. If true, context-dependent retrieval of VOR adaptation should depend on the duration, frequency, and consistency of VR engagement. For instance, in one study, VOR adaptation paired with a unique head orientation was retained for a much longer time than the training duration, and some retention existed outside of the training context (Yakushin et al., 2003; Schubert et al., 2008). To understand VR as a context for reflex adaptation, we need to address whether these gains are truly remembered or learned de novo each time, albeit at a faster rate with the help of familiar sensory cues.

\subsection{VR as a Context for Adaptation of Voluntary Movements}

Adaptation of voluntary movements refers to the integration of proprioceptive and visual information of movement outcomes to reduce sensory prediction error by updating an internal model. It is typically studied by examining changes in movement patterns in response to visuoproprioceptive discordance, such as in the prismatic adaptation paradigm (Redding et al., 2005; Luauté et al., 2009; Redding and Wallace, 


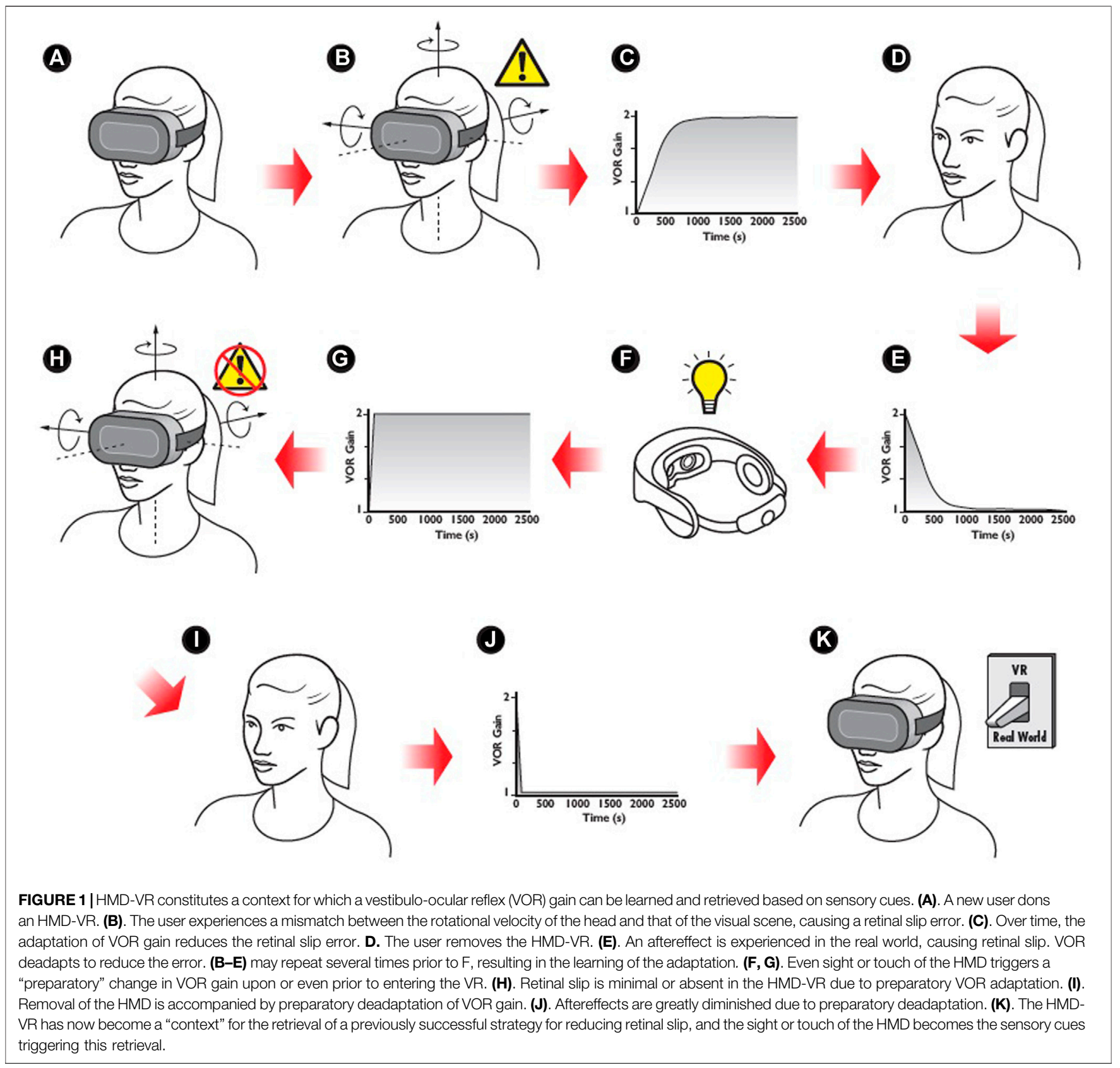

2013). Individuals can learn to toggle between learned adaptations and multiple environments by rapidly retrieving the appropriate internal model or strategy based on specific sensory cues (Mistry and Contreras-Vidal, 2004; Hegele and Heuer, 2010; Huberdeau et al., 2015; Schween et al., 2018). Errors in co-registration between the head and virtual scene can cause displacement and rotation of the virtual display with respect to the real world, inducing visual-proprioceptive discordance (Draper, 1996). Visual-proprioceptive discordance may also arise from body tracking errors resulting in displacements or gains between real-world movements and those of virtual avatars (Draper, 1996).
Accumulating evidence suggests that VR might encourage reliance on explicit learning strategies based on explicit knowledge about the task and target error (Taylor et al., 2014; Taylor and Ivry, 2014), in contrast to implicit adaptation, or "error-based learning," which improves performance continuously and involves updating an internal model based on sensory prediction errors. Researchers evaluated differences in motor learning mechanisms between a 2D screen-based visuomotor adaptation task and HMD-VR presentation of the same task (Anglin et al., 2017). Participants were more likely to use explicit strategies in HMD-VR, although in both conditions, they required the same time to adapt to the perturbation and 
reduce errors. In another study, participants showed larger aftereffects in a prismatic adaptation task in HMD-VR compared to prism goggles (Ramos et al., 2019).

Evaluating aftereffects is critical to learning in VR, but few studies have investigated this variable. Findings from the real world indicate that explicit strategies provide rapid performance improvements during adaptation and are particularly beneficial for tasks requiring rapid and precise mastery of a visuomotor transformation. However, explicit strategies may be detrimental in tasks requiring consistent performance during learning (e.g., performing an endoscopic surgery using a robotic device).

Explicit strategies are predominantly used early in the learning process, while later learning relies on the adoption and use of an internal model (Taylor and Ivry, 2011; Taylor et al., 2014; McDougle et al., 2015). Studies that include a focus on duration of VR engagement are especially important to understanding if, and at what point, individuals adapt an implicit strategy in VR. Additionally, the nature of the learning process is a critical factor for understanding the transfer of skills from VR to the real world. VR-based learning often shows little transfer to the real world (Levac et al., 2019). For instance, amplification of errors in VR negatively impacted transfer due to the use of different coordination strategies (Marchal-Crespo et al., 2017, 2019). Even in older adults and healthy controls, practice in VR does not transfer to the real world (de Mello Monteiro et al., 2014). The typical approach to enhancing skill transfer is to increase the similarity between virtual and real tasks (Levac et al., 2019). However, this attempt would be useless if the brain perceives $\mathrm{VR}$ as a distinct context.

\subsection{VR as a Context for Spatial Navigation}

Navigation involves the use of 1) idiothetic or "self-motion" cues (e.g., vestibular, proprioceptive, efference) generated by the body and head movements for multisensory path integration and 2) allothetic or "landmark" cues (e.g., visual, auditory, tactile) for processing landmark information. Integration of the two is necessary to specify an individual's spatial orientation in allocentric coordinates. The entailed visual, proprioceptive, and vestibular multisensory integration might differ between VR and the real world.

The importance of self-motion in spatial navigation is well demonstrated. The accuracy of scene recognition is reduced when an array of objects is rotated relative to a stationary observer but not when the observer moves relative to a stationary display (Simons and Wang, 1998). Self-motion, but not the passive motion of objects, facilitates scene recognition from novel viewpoints (Witmer and Kline, 1998; Wang and Simons, 1999), and self-motion is critical for orientation (Klatzky et al., 1998). Not surprisingly, given their susceptibility to disorientation after visual rotations, people face difficulty in learning spatial layouts in VR (Richardson et al., 1999). Context-specific learning in VR does not necessarily involve bodily self-movement in the visual scene (Riecke et al., 2010), but spatial navigation within VR may entail intrinsic conflicts due to a false sense of motion induced by optic flow (Park et al., 2018).
The distinct relationship between self-motion and optic flow in VR likely leads to distinct ways in which spatial information is encoded (Aghajan et al., 2015).

In summary, navigation in VR likely does not engage the idiothetic component of "self-motion" comparable to that in the real world. It is immensely challenging to fully identify how this fact influences how VR is interpreted by the brain as a unique context.

\section{KEY FACTORS THAT INFLUENCE THE INTERPRETATION OF VR AS A SENSORIMOTOR CONTEXT}

\subsection{Technological Factors}

Sensory conflict in HMD-VR arises from 1) head motion tracking errors, 2) body motion tracking errors, and 3) delays, lags, and errors in optic flow (Figure 2) (Holloway, 1995). The previous sections have described the processes by which tracking errors lead to sensory adaptations with a focus on dynamic head-tracking errors and VOR adaptation, static head or body tracking errors and adaptation of voluntary arm movements, and the influence of optic flow errors on spatial navigation. Currently, little is known about how the type, magnitude, and variability of VR-system errors affect the adaptation and recall of sensorimotor representations. Information about these factors is critical to engineering innovation in VR to further decrease the gap between the real and virtual world. In this regard, "presence" becomes a critical lens through which to view these factors that determine the extent to which the brain interprets VR as a distinct context. Presence most broadly refers to "the perceived realness of a mediated or virtual experience" (Skarbez et al., 2017). However, "presence" as a universal construct for evaluating VR remains amorphous (see Skarbez et al., 2017 for an in-depth discussion of the definitions of presence). Several definitions of presence concentrate on sensorimotor coupling in the virtual world (Slater and Wilbur, 1997; Zahorik and Jenison, 1998; Slater, 2009; Skarbez et al., 2017), with perhaps the most well established being Slater's "response-asif-real (RAIR)" formulation (Slater, 2009). RAIR states that if a VR user experiences Place Illusion (sense of being in the virtual environment) and Plausibility Illusion (the sense that the virtual experience is really happening), then they should react to virtual stimuli as if they were real. Place Illusion is described to be a function of the sensorimotor contingencies, referred to as immersion, afforded by the virtual reality system. In contrast, Plausibility Illusion is described to be a function of the internal logical and behavioral consistency, referred to as coherence, of the virtual experience. Importantly, this formulation of presence can be assessed objectively through measurements of participant behavior and is, therefore, most relevant to the notion that VR may represent a context for adaptation. We note that this is indeed distinct from definitions of presence that describe "feeling" present, which is a subjective response most often measured by self-report. Whether the sense of 


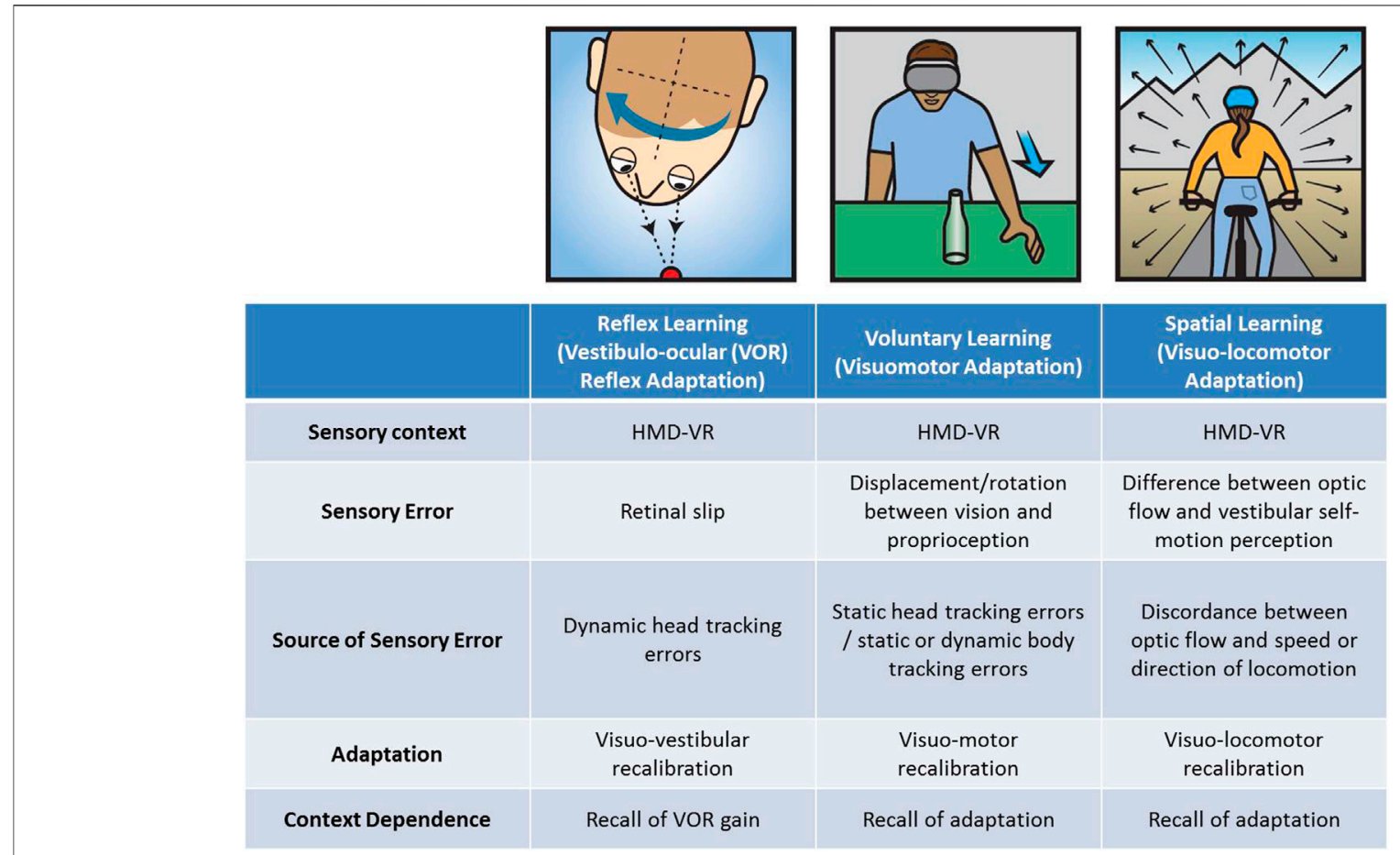

FIGURE 2 | Three examples of context-specific learning in head-mounted display-based virtual reality (HMD-VR).

presence in VR is related to the extent to which VR is a context for sensorimotor learning remains an open question that may add an important new dimension to the study of presence.

\subsection{Human Factors}

Age is a critical factor influencing the extent to which VR is interpreted as a distinct context. VOR gain changes in early development ( $<10$ years) have been linked to the development of inhibitory control of the reticular formation in the brainstem (Ornitz et al., 1985). VOR gain also reduces in aging individuals ( $>75$ years) (Baloh et al., 1993), indicating a reduction in reflex adaptation. Due to poorly calibrated VOR in these populations, head movements can cause image motion on the retina, leading to deficits in motor learning in VR. Whether VR is associated with greater sensitivity to retinal slip and whether VOR adaptation, retention, and consolidation in VR proceed the same way over the lifespan remain open questions.

Young children may experience VR as real to a greater extent than adults do (Flavell et al., 1990; Sharar et al., 2007; Richert et al., 2011; Bailey and Bailenson, 2017) and even respond to non-immersive virtual environments in a way that is cognitively and behaviorally distinct from adults (Baumgartner et al., 2006, 2008). In two studies, adolescents (13-17 years of age) (Baumgartner et al., 2006) and adults (21-43 years of age) (Baumgartner et al., 2006) were found to recruit the prefrontal cortex during the virtual engagement more than children (8-11 years old and 6-11 years old, respectively). It may be that young children, who have a less mature prefrontal cortex and feel more presence in virtual environments, might show increased reliance on implicit learning strategies and may consequently experience a greater degree of interference between realworld tasks and VR. Indeed, evidence indicates that VR might interfere with the normal development of sensorimotor coordination (Miehlbradt et al., 2020) due to an increased reliance on the information obtained from the modality with the highest context-dependent reliability (Gori et al., 2008; Nardini et al., 2014). However, we are unaware of systematic investigations about the sensorimotor consequences of prolonged VR engagement in pediatric populations.

In contrast to the younger populations, aging increases reliance on sensorimotor predictions about the consequences of self-generated actions due to the structural and functional changes in frontostriatal circuits (Wolpe et al., 2016). Older populations may therefore be more likely than young adults to interpret VR as a distinct context. These agerelated changes are important to consider since they may make VR-based training less likely to transfer to the real world in these geriatric populations, as has been reported (Levac et al., 2019). Beyond age, sensorimotor deficits due to various health conditions might also affect the scope of VR-based interventions in clinical populations, though conclusive evidence remains sparse. Finally, other human factors to consider include sex-related differences. In fact, sex-related differences in postural stability in VR have been noted in the literature; women are more likely than men to experience 
cybersickness in VR (Koslucher et al., 2016; Munafo et al., 2017). Additional studies should examine whether these differences percolate to reflex adaptation, the adaptation of voluntary movements, and spatial navigation and if the female brain interprets VR as a distinct context more readily than the male brain does.

Overall, an emerging theme is that the developmental status of the prefrontal cortex (young children), and the ability to integrate multisensory information quickly and veridically (aging adults), influences the extent to which the brain interprets $\mathrm{VR}$ as a distinct context, and the sense of presence may be the critical component mediating its influence on cognition and behavior.

\subsection{Duration of VR Engagement}

Most investigations of sensory conflict in VR involve a single session with less than 2 hours of VR engagement. Even these studies have been limited to subjective reports of cybersickness caused by visuovestibular conflict (Gallagher and Ferrè, 2018; Weech et al., 2018; Kim et al., 2020). Evidence that increased duration of single-session VR engagement increases self-reported cybersickness (Kennedy et al., 2000; Kourtesis et al., 2019) and repeated exposure to VR reduces self-reported cybersickness (Kennedy et al., 2000; Risi and Palmisano, 2019) offers insights into how the duration and frequency of VR engagement might be related to context-dependent learning. In particular, reduced cybersickness with repeated VR engagement might indicate a strategy to overcome sensory conflict errors learned during previous VR engagements. This hypothesis is also in line with recent reports that faster readaptation to a learned sensory conflict relies more on retrieving explicit learning than faster implicit learning (Avraham et al., 2021).

When sensory conflict resolution in VR is viewed as a form of context-dependent learning, exciting questions emerge about how the schedule of VR engagement affects known properties of context-dependent learning. What schedule of engagement is required for VR to constitute a contextual cue for retrieval of learned adaptations? Certain types of contextdependent learning, such as fear conditioning, form strong context-dependent memories upon a single exposure to the context (Maren et al., 2013; Lonsdorf et al., 2017), whereas other types of learning require repeated context-dependent learning to form strong context-dependent memories (Ingram et al., 2011; Ruitenberg et al., 2012; Lee and Fisher, 2019). It is important to understand the interaction between the strength of context-dependent memories of learned adaptations and variability in the magnitude of sensory conflict upon repeated VR engagement. If tracking errors or visual display lags vary even slightly, retrieving a learned adaptation may interfere with the recalibration of sensory adaptations (Fu and Santello, 2012). Probing the effects of different forms of interference on context encoding, conditioning, retrieval, and extinction would provide valuable information about how VR-induced sensory conflict is resolved (Bouton, 1994, 2010).

\section{DISCUSSION}

Understanding the extent to which the brain interprets VR as a unique context precludes sustained and successful adoption of VR technology. Context-dependent learning may either be an asset or a hindrance to VR engagements. When used for entertainment, teleconferencing, or work it may be preferable to minimize carryover of sensorimotor adaptations from VR to the real world. Because short-lasting or absent aftereffects are a hallmark of context-dependent learning, it may be desirable to enhance context dependency of learned adaptations for these use cases. In contrast, when VR is used for for skills training or rehabilitation it may be desirable to reduce the contextdependency of learning to enhance aftereffects and ultimately generalization of learning from VR to the real world. This transfer might be accomplished by reducing the repeatability of the environment or increasing the presence of the experience.

A complete absence of visuomotor discrepancies, or full immersion, has been previously hypothesized to give rise to a strong sense of Place Illusion (Skarbez et al., 2017; Slater, 2009). Given the definition of context presented here, the complete absence of visuomotor discrepancies would theoretically remove the need for interpreting $\mathrm{VR}$ as a context for sensorimotor adaption. However, whether this is true or not remains to be tested. Perhaps the more pertinent question is, how veridical does a VR system need to be to remove context? Furthermore, it is likely that the magnitude and type of sensorimotor discordance may affect context dependencies of reflex conditioning, voluntary motor adaptation, and spatial navigation differently. We hypothesize that VR as a context for spatial navigation is likely distinct from VR as a context for reflex adaptation or for adaptation of voluntary movements. Low coherence within the virtual world, yielding poor Plausibility Illusion, is more likely to influence how spatial information is encoded and may likely constitute a context distinct from the real world that persists even when sensorimotor discordance is low. Studies that address the technological and human factors that may influence whether the brain interprets a context distinct from the real world are few and far between. The following questions remain open for both the engineers developing the systems and the perceptual scientists interested in the neurological effects of VR:

- How do the duration, frequency, and schedule of engagement influence whether the brain perceives VR as a context distinct from the real world?

- What are the aftereffects of VR engagement and how do they change with repeated exposure?

- What are the thresholds for sensorimotor adaptation in VR? How close to the human perceptual threshold can sensory conflicts occur without causing the individual to invoke a learning or adaptation strategy? Does it matter if sensory conflicts occur suddenly or gradually?

- How do sensory conflicts arising from multiple sources of error (e.g., head and body tracking errors combined) affect adaptation? Is there a different threshold for each source of error? 
- Are there interference or reinforcement effects between training performed in VR and transferred to the real world?

Future work on context-dependent learning based on numerous well-validated designs previously used for testing retrieval, interference, and savings (Krakauer et al., 1999, 2005; Zarahn et al., 2008; Huang et al., 2011; Taylor et al., 2014) can provide a greater understanding of the extent to which the brain interprets VR as a unique context, providing invaluable information to VR applications across multiple domains.

\section{DATA AVAILABILITY STATEMENT}

No original data was included in the article, further inquiries can be directed to the corresponding authors.

\section{REFERENCES}

Affouneh, S., Salha, S., and Khlaif, Z. N. (2020). Designing Quality E-Learning Environments for Emergency Remote Teaching in Coronavirus Crisis. Interdiscip. J. Virtual Learn. Med. Sci. 11, 135-137.

Aghajan, Z. M., Acharya, L., Moore, J. J., Cushman, J. D., Vuong, C., and Mehta, M. R. (2015). Impaired Spatial Selectivity and Intact Phase Precession in TwoDimensional Virtual Reality. Nat. Neurosci. 18, 121-128. doi:10.1038/nn.3884

Aiba, A., Chen, C., Herrup, K., Rosenmund, C., Stevens, C. F., and Tonegawa, S. (1994). Reduced Hippocampal Long-Term Potentiation and Context-specific Deficit in Associative Learning in Mglur1 Mutant Mice. Cell 79, 365-375. doi:10.1016/0092-8674(94)90204-6

Anglin, J. M., Sugiyama, T., and Liew, S. L. (2017). Visuomotor Adaptation in Head-Mounted Virtual Reality versus Conventional Training. Sci. Rep. 7, 45469-45478. doi:10.1038/srep45469

Antoniadis, E., and McDonald, R. J. (2000). Amygdala, hippocampus and Discriminative Fear Conditioning to Context. Behav. Brain Res. 108, 1-19. doi:10.1016/s0166-4328(99)00121-7

Avraham, G., Morehead, J. R., Kim, H. E., and Ivry, R. B. (2021). Reexposure to a Sensorimotor Perturbation Produces Opposite Effects on Explicit and Implicit Learning Processes. Plos Biol. 19, e3001147. doi:10.1371/journal.pbio.3001147

Bailey, J. O., and Bailenson, J. N. (2017). "Immersive Virtual Reality and the Developing Child," in Cognitive Development in Digital Contexts. Elsevier, 181-200. doi:10.1016/b978-0-12-809481-5.00009-2

Baloh, R. W., Jacobson, K. M., and Socotch, T. M. (1993). The Effect of Aging on VisualVestibuloocular Responses. Exp. Brain Res. 95, 509-516. doi:10.1007/BF00227144

Baumgartner, T., Speck, D., Wettstein, D., Masnari, O., Beeli, G., and Jäncke, L. (2008). Feeling Present in Arousing Virtual Reality Worlds: Prefrontal Brain Regions Differentially Orchestrate Presence Experience in Adults and Children. Front. Hum. Neurosci. 2, 8. doi:10.3389/neuro.09.008.2008

Baumgartner, T., Valko, L., Esslen, M., and Jäncke, L. (2006). Neural Correlate of Spatial Presence in an Arousing and Noninteractive Virtual Reality: An Eeg and Psychophysiology Study. CyberPsychology Behav. 9, 30-45. doi:10.1089/ cpb.2006.9.30

Bouton, M. E. (1994). Context, Ambiguity, and Classical Conditioning. Curr. Dir. Psychol. Sci. 3, 49-53. doi:10.1111/1467-8721.ep10769943

Bouton, M. E. (2010). "The Multiple Forms of "context" in Associative Learning Theory," in The Mind in Context. Editors B. Mesquita, L. F. Barrett, and E. R. Smith (New York: Guilford Press), 233-258.

Burguière, E., Arleo, A., Hojjati, M. r., Elgersma, Y., Zeeuw, C. I. D., Berthoz, A., et al. (2005). Spatial Navigation Impairment in Mice Lacking Cerebellar Ltd: A Motor Adaptation Deficit? Nat. Neurosci. 8, 1292-1294. doi:10.1038/nn1532

Collewijn, H., Martins, A. J., and Steinman, R. M. (1983). Compensatory Eye Movements during Active and Passive Head Movements: Fast Adaptation to Changes in Visual Magnification. J. Physiol. 340, 259-286. doi:10.1113/ jphysiol.1983.sp014762

\section{AUTHOR CONTRIBUTIONS}

MY, MM, SN, and ET conceived the idea for the manuscript; MY, $\mathrm{MM}$, and SN drafted the manuscript; MY, MM, SN, and ET edited and revised manuscript; MY, MM, SN, and ET approved the final version of manuscript.

\section{FUNDING}

The project was primarily funded by a research contract under Facebook's Sponsored Academic Research Agreement. The project was additionally supported in part by $\mathrm{NIH}$ 2R01NS085122 (ET), NIH-2R01HD058301 (ET), NSFCBET-1804550 (ET), and NSF-CMMI-M3X-1935337 (ET and MY)

de Mello Monteiro, C. B., Massetti, T., da Silva, T. D., van der Kamp, J., de Abreu, L. C., Leone, C., et al. (2014). Transfer of Motor Learning from Virtual to Natural Environments in Individuals with Cerebral Palsy. Res. Develop. Disabilities 35, 2430-2437. doi:10.1016/j.ridd.2014.06.006

De Ponti, R., Marazzato, J., Maresca, A. M., Rovera, F., Carcano, G., and Ferrario, M. M. (2020). Pre-graduation Medical Training Including Virtual Reality during COVID-19 Pandemic: a Report on Students' Perception. BMC Med. Educ. 20, 332-337. doi:10.1186/s12909-020-02245-8

Demer, J. L., Goldberg, J., Jenkins, H. A., and Porter, F. I. (1987). Vestibulo-ocular Reflex during Magnified Vision: Adaptation to Reduce Visual-Vestibular Conflict. Aviat Space Environ. Med. 58, A175-A179.

DiZio, P., and Lackner, J. R. (1992). Spatial Orientation, Adaptation, and Motion Sickness in Real and Virtual Environments. Presence: Teleoperators \& Virtual Environments 1, 319-328. doi:10.1162/pres.1992.1.3.319

Draper, M. H. (1996). Can Your Eyes Make You Sick? Investigating the Relationship between the Vestibulo-Ocular Reflex and Virtual Reality. Human Interface Technology Laboratory Technical Report.

Draper, M. H. (1998). The Adaptive Effects of Virtual Interfaces: Vestibulo-Ocular Reflex and Simulator Sickness. Ph.D. Thesis. Air Force Institute of Technology at Wright-Patterson AFB.

Ehrlich, H., McKenney, M., and Elkbuli, A. (2020). We Asked the Experts: Virtual Learning in Surgical Education during the COVID-19 Pandemic-Shaping the Future of Surgical Education and Training. World J. Surg. 44, 2053-2055. doi:10.1007/s00268-020-05574-3

Federmeier, K. D., McLennan, D. B., Ochoa, E., and Kutas, M. (2002). The Impact of Semantic Memory Organization and Sentence Context Information on Spoken Language Processing by Younger and Older Adults: An Erp Study. Psychophysiology 39, 133-146. doi:10.1111/1469-8986.3920133

Flavell, J. H., Flavell, E. R., Green, F. L., and Korfmacher, J. E. (1990). Do young Children Think of Television Images as Pictures or Real Objects? J. Broadcasting Electron. Media 34, 399-419. doi:10.1080/08838159009386752

Frances Wang, R., and Simons, D. J. (1999). Active and Passive Scene Recognition across Views. Cognition 70, 191-210. doi:10.1016/s0010-0277(99)00012-8

$\mathrm{Fu}, \mathrm{Q}$, and Santello, M. (2012). Context-dependent Learning Interferes with Visuomotor Transformations for Manipulation Planning. J. Neurosci. 32, 15086-15092. doi:10.1523/jneurosci.2468-12.2012

Gallagher, M., and Ferrè, E. R. (2018). Cybersickness: A Multisensory Integration Perspective. Multisens. Res. 31, 645-674. doi:10.1163/22134808-20181293

Gauthier, G. M., and Robinson, D. A. (1975). Adaptation of the Human Vestibuloocular Reflex to Magnifying Lenses. Brain Res. 92, 331-335. doi:10.1016/0006-8993(75)90279-6

Gimmon, Y., Migliaccio, A. A., Todd, C. J., Figtree, W. V. C., and Schubert, M. C. (2018). Simultaneous and opposing horizontal vor adaptation in humans suggests functionally independent neural circuits. J. Neurophysiol. 120, 1496-1504. doi:10.1152/jn.00134.2018

Glover, S., and Dixon, P. (2001). Motor Adaptation to an Optical Illusion. Exp. Brain Res. 137, 254-258. doi:10.1007/s002210000651 
Gonshor, A., and Jones, G. M. (1976a). Extreme Vestibulo-Ocular Adaptation Induced by Prolonged Optical Reversal of Vision. J. Physiol. 256, 381-414. doi:10.1113/jphysiol.1976.sp011330

Gonshor, A., and Jones, G. M. (1976b). Short-term Adaptive Changes in the Human Vestibulo-Ocular Reflex Arc. J. Physiol. 256, 361-379. doi:10.1113/ jphysiol.1976.sp011329

Gori, M., Del Viva, M., Sandini, G., and Burr, D. C. (2008). Young Children Do Not Integrate Visual and Haptic Form Information. Curr. Biol. 18, 694-698. doi:10.1016/j.cub.2008.04.036

Hegele, M., and Heuer, H. (2010). Implicit and Explicit Components of Dual Adaptation to Visuomotor Rotations. Conscious. Cogn. 19, 906-917. doi:10.1016/j.concog.2010.05.005

Herdman, S. J. (1998). Role of Vestibular Adaptation in Vestibular Rehabilitation. Otolaryngol. Head Neck Surg. 119, 49-54. doi:10.1016/s0194-5998(98)70195-0

Holloway, R. L. (1995). Registration Errors in Augmented Reality Systems. Ph.D. thesis, University of North Carolina at Chapel Hill.

Huang, V. S., Haith, A., Mazzoni, P., and Krakauer, J. W. (2011). Rethinking Motor Learning and Savings in Adaptation Paradigms: Model-free Memory for Successful Actions Combines with Internal Models. Neuron 70, 787-801. doi:10.1016/j.neuron.2011.04.012

Huberdeau, D. M., Haith, A. M., and Krakauer, J. W. (2015). Formation of a LongTerm Memory for Visuomotor Adaptation Following Only a Few Trials of Practice. J. Neurophysiol. 114, 969-977. doi:10.1152/jn.00369.2015

Ingram, J. N., Howard, I. S., Flanagan, J. R., and Wolpert, D. M. (2011). A Single-Rate Context-dependent Learning Process Underlies Rapid Adaptation to Familiar Object Dynamics. Plos Comput. Biol. 7, e1002196. doi:10.1371/journal.pcbi.1002196

Ito, M. (1998). Cerebellar Learning in the Vestibulo-Ocular Reflex. Trends Cogn. Sci. 2, 313-321. doi:10.1016/s1364-6613(98)01222-4

Jones, M. N., Willits, J., Dennis, S., and Jones, M. (2015). Models of Semantic Memory. Oxford Handbook Math. Comput. Psychol., 232-254. doi:10.1093/ oxfordhb/9780199957996.013.11

Kennedy, R. S., Stanney, K. M., and Dunlap, W. P. (2000). Duration and Exposure to Virtual Environments: Sickness Curves during and across Sessions. Presence: Teleoperators \& Virtual Environments 9, 463-472. doi:10.1162/105474600566952

Kim, J., Luu, W., and Palmisano, S. (2020). Multisensory Integration and the Experience of Scene Instability, Presence and Cybersickness in Virtual Environments. Comput. Hum. Behav. 113, 106484. doi:10.1016/j.chb.2020.106484

Klatzky, R. L., Loomis, J. M., Beall, A. C., Chance, S. S., and Golledge, R. G. (1998). Spatial Updating of Self-Position and Orientation during Real, Imagined, and Virtual Locomotion. Psychol. Sci. 9, 293-298. doi:10.1111/1467-9280.00058

Koslucher, F., Haaland, E., and Stoffregen, T. A. (2016). Sex Differences in Visual Performance and Postural Sway Precede Sex Differences in Visually Induced Motion Sickness. Exp. Brain Res. 234, 313-322. doi:10.1007/s00221-015-4462-y

Kourtesis, P., Collina, S., Doumas, L. A. A., and MacPherson, S. E. (2019). Validation of the Virtual Reality Neuroscience Questionnaire: Maximum Duration of Immersive Virtual Reality Sessions without the Presence of Pertinent Adverse Symptomatology. Front. Hum. Neurosci. 13, 417. doi:10.3389/fnhum.2019.00417

Krakauer, J. W., Ghez, C., and Ghilardi, M. F. (2005). Adaptation to Visuomotor Transformations: Consolidation, Interference, and Forgetting. J. Neurosci. 25, 473-478. doi:10.1523/jneurosci.4218-04.2005

Krakauer, J. W., Ghilardi, M.-F., and Ghez, C. (1999). Independent Learning of Internal Models for Kinematic and Dynamic Control of Reaching. Nat. Neurosci. 2, 1026-1031. doi:10.1038/14826

Kramida, G. (2016). Resolving the Vergence-Accommodation Conflict in HeadMounted Displays. IEEE Trans. Vis. Comput. Graph 22, 1912-1931. doi:10.1109/TVCG.2015.2473855

Kutas, M., and Federmeier, K. D. (2000). Electrophysiology Reveals Semantic Memory Use in Language Comprehension. Trends Cogn. Sci. 4, 463-470. doi:10.1016/s1364-6613(00)01560-6

Lee, Y.-Y., and Fisher, B. E. (2019). The Effect of Practice Schedule on Context-dependent Learning. J. Mot. Behav. 51, 121-128. doi:10.1080/00222895.2018.1437020

Levac, D. E., Huber, M. E., and Sternad, D. (2019). Learning and Transfer of Complex Motor Skills in Virtual Reality: A Perspective Review. J. Neuroeng Rehabil. 16, 121-215. doi:10.1186/s12984-019-0587-8

Lewis, R. F., Clendaniel, R. A., and Zee, D. S. (2003). Vergence-dependent Adaptation of the Vestibulo-Ocular Reflex. Exp. Brain Res. 152, 335-340. doi:10.1007/s00221-003-1563-9
Lonsdorf, T. B., Menz, M. M., Andreatta, M., Fullana, M. A., Golkar, A., Haaker, J., et al. (2017). Don't Fear 'fear Conditioning': Methodological Considerations for the Design and Analysis of Studies on Human Fear Acquisition, Extinction, and Return of Fear. Neurosci. Biobehavioral Rev. 77, 247-285. doi:10.1016/ j.neubiorev.2017.02.026

Luauté, J., Schwartz, S., Rossetti, Y., Spiridon, M., Rode, G., Boisson, D., et al. (2009). Dynamic Changes in Brain Activity during Prism Adaptation. J. Neurosci. 29, 169-178. doi:10.1523/jneurosci.3054-08.2009

Mantovani, E., Zucchella, C., Bottiroli, S., Federico, A., Giugno, R., Sandrini, G., et al. (2020). Telemedicine and Virtual Reality for Cognitive Rehabilitation: A Roadmap for the Covid-19 Pandemic. Front. Neurol. 11, 926. doi:10.3389/ fneur.2020.00926

Marchal-Crespo, L., Michels, L., Jaeger, L., López-Olóriz, J., and Riener, R. (2017). Effect of Error Augmentation on Brain Activation and Motor Learning of a Complex Locomotor Task. Front. Neurosci. 11, 526. doi:10.3389/fnins.2017.00526

Marchal-Crespo, L., Tsangaridis, P., Obwegeser, D., Maggioni, S., and Riener, R. (2019). Haptic Error Modulation Outperforms Visual Error Amplification when Learning a Modified Gait Pattern. Front. Neurosci. 13, 61. doi:10.3389/fnins.2019.00061

Maren, S., Phan, K. L., and Liberzon, I. (2013). The Contextual Brain: Implications for Fear Conditioning, Extinction and Psychopathology. Nat. Rev. Neurosci. 14, 417-428. doi:10.1038/nrn3492

Marschner, A., Kalisch, R., Vervliet, B., Vansteenwegen, D., and Büchel, C. (2008). Dissociable Roles for the hippocampus and the Amygdala in Human Cued versus Context Fear Conditioning. J. Neurosci. 28, 9030-9036. doi:10.1523/ jneurosci.1651-08.2008

McDougle, S. D., Bond, K. M., and Taylor, J. A. (2015). Explicit and Implicit Processes Constitute the Fast and Slow Processes of Sensorimotor Learning. J. Neurosci. 35, 9568-9579. doi:10.1523/jneurosci.5061-14.2015

Micarelli, A., Viziano, A., Augimeri, I., Micarelli, D., and Alessandrini, M. (2017). Three-dimensional Head-Mounted Gaming Task Procedure Maximizes Effects of Vestibular Rehabilitation in Unilateral Vestibular Hypofunction: A Randomized Controlled Pilot Trial. Int. J. Rehabil. Res. 40, 325-332. doi: 10.1097/mrr.0000000000000244

Micarelli, A., Viziano, A., Micarelli, B., Augimeri, I., and Alessandrini, M. (2019). Vestibular Rehabilitation in Older Adults with and without Mild Cognitive Impairment: Effects of Virtual Reality Using a Head-Mounted Display. Arch. Gerontol. Geriatr. 83, 246-256. doi:10.1016/j.archger.2019.05.008

Miehlbradt, J. C., Cuturi, L. F., Zanchi, S., Gori, M., and Micera, S. (2020). Immersive Virtual Reality Interferes with Default Head-Trunk Coordination Strategies in Young Children. bioRxiv.

Mistry, S., and Contreras-Vidal, J. L. (2004). Learning Multiple Visuomotor Transformations: Adaptation and Context-dependent Recall. Motor Control 8, 534-546. doi:10.1123/mcj.8.4.534

Munafo, J., Diedrick, M., and Stoffregen, T. A. (2017). The Virtual Reality HeadMounted Display Oculus Rift Induces Motion Sickness and Is Sexist in its Effects. Exp. Brain Res. 235, 889-901. doi:10.1007/s00221-016-4846-7

Nardini, M., Dekker, T., and Petrini, K. (2014). Crossmodal Integration: a Glimpse into the Development of Sensory Remapping. Curr. Biol. 24, R532-R534. doi:10.1016/j.cub.2014.04.034

Neszmélyi, B., and Horváth, J. (2019). The Role of Auditory Context in ActionEffect-Related Motor Adaptation. Hum. Move. Sci. 67, 102503. doi:10.1016/ j.humov.2019.102503

Ornitz, E. M., Kaplan, A. R., and Westlake, J. R. (1985). Development of the Vestibulo-Ocular Reflex from Infancy to Adulthood. Acta Oto-Laryngologica 100, 180-193. doi:10.3109/00016488509104780

Paige, G. D., and Sargent, E. W. (1991). Visually-induced Adaptive Plasticity in the Human Vestibulo-Ocular Reflex. Exp. Brain Res. 84, 25-34. doi:10.1007/ BF00231759

Park, J. L., Dudchenko, P. A., and Donaldson, D. I. (2018). Navigation in RealWorld Environments: New Opportunities Afforded by Advances in mobile Brain Imaging. Front. Hum. Neurosci. 12, 361. doi:10.3389/ fnhum.2018.00361

Pears, M., Yiasemidou, M., Ismail, M. A., Veneziano, D., and Biyani, C. S. (2020). Role of Immersive Technologies in Healthcare Education during the Covid-19 Epidemic. Scott Med. J. 65, 112-119. doi:10.1177/0036933020956317

Pregowska, A., Masztalerz, K., Garlińska, M., and Osial, M. (2021). A Worldwide Journey through Distance Education-From the Post Office to Virtual, 
Augmented and Mixed Realities, and Education during the COVID-19 Pandemic. Edu. Sci. 11, 118. doi:10.3390/educsci11030118

Ramos, A. A., Hørning, E. C., and Wilms, I. L. (2019). Simulated Prism Exposure in Immersed Virtual Reality Produces Larger Prismatic After-Effects Than Standard Prism Exposure in Healthy Subjects. PLoS One 14, e0217074. doi:10.1371/journal.pone.0217074

Redding, G. M., Rossetti, Y., and Wallace, B. (2005). Applications of Prism Adaptation: A Tutorial in Theory and Method. Neurosci. Biobehavioral Rev. 29, 431-444. doi:10.1016/j.neubiorev.2004.12.004

Redding, G. M., and Wallace, B. (2013). Prism Adaptation in Alternately Exposed Hands. Atten Percept Psychophys 75, 1168-1185. doi:10.3758/s13414-013-0467-4

Richardson, A. E., Montello, D. R., and Hegarty, M. (1999). Spatial Knowledge Acquisition from Maps and from Navigation in Real and Virtual Environments. Mem. Cogn. 27, 741-750. doi:10.3758/bf03211566

Richert, R. A., Robb, M. B., and Smith, E. I. (2011). Media as Social Partners: The Social Nature of Young Children's Learning from Screen Media. Child. Dev. 82, 82-95. doi:10.1111/j.1467-8624.2010.01542.x

Richter, S., Jansen-Osmann, P., Konczak, J., and Kalveram, K. T. (2004). Motor Adaptation to Different Dynamic Environments Is Facilitated by Indicative Context Stimuli. Psychol. Res. 68, 245-251. doi:10.1007/s00426-003-0140-y

Riecke, B. E., Bodenheimer, B., McNamara, T. P., Williams, B., Peng, P., and Feuereissen, D. (2010). "Do we Need to Walk for Effective Virtual Reality Navigation? Physical Rotations Alone May Suffice," in International Conference on Spatial Cognition (Springer), 234-247. doi:10.1007/978-3-642-14749-4_21

Risi, D., and Palmisano, S. (2019). Effects of Postural Stability, Active Control, Exposure Duration and Repeated Exposures on Hmd Induced Cybersickness. Displays 60, 9-17. doi:10.1016/j.displa.2019.08.003

Rosas, J. M., Todd, T. P., and Bouton, M. E. (2013). Context Change and Associative Learning. Wires Cogn. Sci. 4, 237-244. doi:10.1002/wcs.1225

Ruitenberg, M. F. L., De Kleine, E., Van der Lubbe, R. H. J., Verwey, W. B., and Abrahamse, E. L. (2012). Context-dependent Motor Skill and the Role of Practice. Psychol. Res. 76, 812-820. doi:10.1007/s00426-011-0388-6

Schubert, M. C., Migliaccio, A. A., Clendaniel, R. A., Allak, A., and Carey, J. P. (2008). Mechanism of Dynamic Visual Acuity Recovery with Vestibular Rehabilitation. Arch. Phys. Med. Rehabil. 89, 500-507. doi:10.1016/ j.apmr.2007.11.010

Schween, R., Taylor, J. A., and Hegele, M. (2018). Plan-based Generalization Shapes Local Implicit Adaptation to Opposing Visuomotor Transformations. J. Neurophysiol. 120, 2775-2787. doi:10.1152/jn.00451.2018

Sharar, S. R., Carrougher, G. J., Nakamura, D., Hoffman, H. G., Blough, D. K., and Patterson, D. R. (2007). Factors Influencing the Efficacy of Virtual Reality Distraction Analgesia during Postburn Physical Therapy: Preliminary Results from 3 Ongoing Studies. Arch. Phys. Med. Rehabil. 88, S43-S49. doi:10.1016/j.apmr.2007.09.004

Sharoni, Z., Shupak, A., Nachum, Z., Spitzer, O., and Gadoth, N. (2001). Vestibular Findings in Professional Divers. Ann. Otol Rhinol Laryngol. 110, 127-131. doi:10.1177/000348940111000207

Shelhamer, M., Robinson, D. A., and Tan, H. S. (1992). Context-specific Adaptation of the Gain of the Vestibulo-Ocular Reflex in Humans. Ves 2, 89-96. doi:10.3233/ves-1992-2107

Sigala, M. (2020). Tourism and Covid-19: Impacts and Implications for Advancing and Resetting Industry and Research. J. Business Res. 117, 312-321. doi:10.1016/j.jbusres.2020.06.015

Simons, D. J., and Wang, R. F. (1998). Perceiving Real-World Viewpoint Changes. Psychol. Sci. 9, 315-320. doi:10.1111/1467-9280.00062

Singh, R. P., Javaid, M., Kataria, R., Tyagi, M., Haleem, A., and Suman, R. (2020). Significant Applications of Virtual Reality for Covid-19 Pandemic. Diabetes Metab. Syndr. Clin. Res. Rev. 14, 661-664. doi:10.1016/j.dsx.2020.05.011

Skarbez, R., Brooks, Jr., F. P., Jr., and Whitton, M. C. (2018). A Survey of Presence and Related Concepts. ACM Comput. Surv. 50, 1-39. doi:10.1145/3134301

Slater, M. (2009). Place Illusion and Plausibility Can lead to Realistic Behaviour in Immersive Virtual Environments. Phil. Trans. R. Soc. B 364, 3549-3557. doi:10.1098/rstb.2009.0138

Slater, M., and Wilbur, S. (1997). A Framework for Immersive Virtual Environments (Five): Speculations on the Role of Presence in Virtual Environments. Presence: Teleoperators \& Virtual Environments 6, 603-616. doi:10.1162/pres.1997.6.6.603

Stefano Di Girolamo, Pasqualina Pic, S., Picciotti, P., Sergi, B., Di Nardo, W., Paludetti, G., and Ottaviani, F. (2001). Vestibulo-ocular Reflex Modification after Virtual Environment Exposure. Acta Oto-laryngologica 121, 211-215. doi:10.1080/000164801300043541

Tan, H. S., Shelhamer, M., and Zee, D. S. (1992). Effect of Head Orientation and Position on Vestibuloocular Reflex Adaptation. Ann. NY Acad. Sci. 656, 158-165. doi:10.1111/j.1749-6632.1992.tb25206.x

Taylor, J. A., and Ivry, R. B. (2014). Cerebellar and Prefrontal Cortex Contributions to Adaptation, Strategies, and Reinforcement Learning. Prog. Brain Res. 210, 217-253. doi:10.1016/b978-0-444-63356-9.00009-1

Taylor, J. A., and Ivry, R. B. (2011). Flexible Cognitive Strategies during Motor Learning. Plos Comput. Biol. 7, e1001096. doi:10.1371/journal.pcbi.1001096

Taylor, J. A., Krakauer, J. W., and Ivry, R. B. (2014). Explicit and Implicit Contributions to Learning in a Sensorimotor Adaptation Task. J. Neurosci. 34, 3023-3032. doi:10.1523/jneurosci.3619-13.2014

Urcelay, G. P., and Miller, R. R. (2014). The Functions of Contexts in Associative Learning. Behav. Process. 104, 2-12. doi:10.1016/j.beproc.2014.02.008

Vailshery, L. S. (2021). https://www.statista.com/statistics/1017008/united-statesvr-ar-users/. statistica.com.

Virre, E. (1996). Virtual Reality and the Vestibular Apparatus. IEEE Eng. Med. Biol. Mag. 15, 41-43. doi:10.1109/51.486717

Viziano, A., Micarelli, A., Augimeri, I., Micarelli, D., and Alessandrini, M. (2019). Long-term Effects of Vestibular Rehabilitation and Head-Mounted Gaming Task Procedure in Unilateral Vestibular Hypofunction: A 12-month Follow-Up of a Randomized Controlled Trial. Clin. Rehabil. 33, 24-33. doi:10.1177/ 0269215518788598

Wang, S. S. Y., Teo, W. Z. W., Teo, W. Z. Y., and Chai, Y. W. (2020). Virtual Reality as a Bridge in Palliative Care during Covid-19. J. Palliat. Med. 23, 756. doi:10.1089/jpm.2020.0212

Wasserman, E. A., and Miller, R. R. (1997). What's Elementary about Associative Learning? Annu. Rev. Psychol. 48, 573-607. doi:10.1146/annurev.psych.48.1.573

Weech, S., Varghese, J. P., and Barnett-Cowan, M. (2018). Estimating the Sensorimotor Components of Cybersickness. J. Neurophysiol. 120, 2201-2217. doi:10.1152/jn.00477.2018

Welch, T. D. J., and Ting, L. H. (2014). Mechanisms of Motor Adaptation in Reactive Balance Control. PLoS One 9, e96440. doi:10.1371/journal.pone.0096440

Witmer, B. G., and Kline, P. B. (1998). Judging Perceived and Traversed Distance in Virtual Environments. Presence 7, 144-167. doi:10.1162/105474698565640

Wolpe, N., Ingram, J. N., Tsvetanov, K. A., Geerligs, L., Kievit, R. A., Henson, R. N., et al. (2016). Ageing Increases reliance on Sensorimotor Prediction through Structural and Functional Differences in Frontostriatal Circuits. Nat. Commun. 7, 13034-13111. doi:10.1038/ncomms13034

Yakushin, S. B., Palla, A., Haslwanter, T., Bockisch, C. J., and Straumann, D. (2003). Dependence of Adaptation of the Human Vertical Angular Vestibulo-Ocular Reflex on Gravity. Exp. Brain Res. 152, 137-142. doi:10.1007/s00221-0031543-0

Zahorik, P., and Jenison, R. L. (1998). Presence as Being-In-The-World. Presence 7, 78-89. doi:10.1162/105474698565541

Zarahn, E., Weston, G. D., Liang, J., Mazzoni, P., and Krakauer, J. W. (2008) Explaining Savings for Visuomotor Adaptation: Linear Time-Invariant StateSpace Models Are Not Sufficient. J. Neurophysiol. 100, 2537-2548. doi:10.1152/ jn. 90529.2008

Conflict of Interest: The authors declare that the research was conducted in the absence of any commercial or financial relationships that could be construed as a potential conflict of interest.

Publisher's Note: All claims expressed in this article are solely those of the authors and do not necessarily represent those of their affiliated organizations, or those of the publisher, the editors, and the reviewers. Any product that may be evaluated in this article, or claim that may be made by its manufacturer, is not guaranteed or endorsed by the publisher.

Copyright (๑) 2021 Yarossi, Mangalam, Naufel and Tunik. This is an open-access article distributed under the terms of the Creative Commons Attribution License (CC $B Y)$. The use, distribution or reproduction in other forums is permitted, provided the original author(s) and the copyright owner(s) are credited and that the original publication in this journal is cited, in accordance with accepted academic practice. No use, distribution or reproduction is permitted which does not comply with these terms. 